\title{
Otimização do estoque de uma fábrica de bebidas não alcoólicas em Python
}

\author{
Inventory Optimization of a soft drink factory in Python
}

\author{
Breno Prado Franchini Bizerra ${ }^{1 *}$, Vinicius Abrão da Silva Marques ${ }^{2 *}$ \\ *Departamento de Engenharia Mecânica, Universidade Federal do Triângulo Mineiro, Uberaba, MG, Brasil. \\ 1Orcid: 0000-0002-0078-0401 E-mail: breno.bizerra@gmail.com, ${ }^{2}$ Orcid: 0000-0002-5499-0404 E-mail: \\ vinicius.marques@uftm.edu.br
}

\begin{abstract}
RESUMO: Este trabalho tem o intuito de apresentar a utilização de modelagem matemática computacional para otimização em Python do estoque de uma fábrica. O problema é construído em torno da organização da produção de bebidas. A utilização dos algoritmos Scipy.optimize.minimize e Algoritmo Genético (Genetic Algorithm) empregados neste trabalho tem como objetivo otimizar a quantidade de produtos em estoque, levando em conta os diferentes produtos fabricados, a previsão de venda baseada em histórico e a capacidade máxima de produção. Os métodos utilizados são comparados em suas respostas e tempo de processamento. Os resultados mostram que ambos os métodos têm a capacidade de encontrar respostas otimizadas para o problema. O método Genetic Algorithm encontra respostas menos otimizadas para cada produto, mas realiza uma melhor distribuição geral da produção, além de necessitar de maior tempo de processamento para analisar todas as possibilidades. O método Scipy.optimize.minimize encontra uma resposta mais otimizada para a maioria dos produtos, mas não realiza uma distribuição eficiente da produção, além de apresentar um menor tempo de processamento.
\end{abstract}

Palavras-chave: Otimização de Estoque, Previsão de vendas, Python, Scipy, Algoritmo Genético.

ABSTRACT: The aim of the present article is to present computational mathematical modeling using to optimize a company's inventory based on Python. The study concerned soft drink production organization. Algorithms Scipy.optimize.minimize and Genetic Algorithm were herein used to optimize the quantity of products in stock by taking into consideration different manufactured goods, forecast sales based on sales history, and production maximum capacity. The adopted methods were compared to assess their responses and processing time. Based on the results, both methods are capable of giving optimal responses. The Genetic Algorithm method gives lesser optimized answers to each product, but it accounts for better general production distribution, although it needs more processing time to analyze all possibilities. The Scipy.optimize.minimize method provided more optimized answer to most products, but it was not efficient in production distribution, although it presented shorter processing time.

Keywords: Inventory Optimization, Sales forecast, Python, Scipy, Genetic Algorithm. 
Revista Brasileira de Ciência, Tecnologia e Inovação

\section{INTRODUÇÃO}

Com o notável crescimento da produção de bebidas não alcoólicas no Brasil nos últimos anos torna-se necessária a existência de maneiras mais eficientes de organização da produção e maior eficiência do cronograma de funcionamento de maquinários, aumentando a produtividade das indústrias (PAGLIARUSSI; MORABITO; SANTOS, 2016). $\mathrm{O}$ crescimento do número de fábricas de pequeno e médio porte levou a uma maior concorrência e crescente exigência de qualidade na categoria. Mas como estar na frente do mercado? (SEBRAE, 2021).

O emprego de ferramentas computacionais de simulação da produção, sua modelagem e criação de métodos de otimizações matemáticas, permitem que a empresa diminua o seu tempo de setup do maquinário e aumente a eficiência do seu cronograma de produção. Sendo assim, têm-se uma diminuição do custo envolvido no processo de fabricação e consequentemente custo final do produto.

Uma das técnicas mais utilizadas de otimização matemática computacional é o Genetic Algorithm. Trata-se de um processo evolucionário, ou seja, se trabalha com uma população de candidatos (possíveis respostas ao problema) em paralelo, sendo possível a busca de diferentes soluções e com alocação de membros em vários espectros de respostas. Por serem baseados em evoluções biológicas, trabalham com o método "gerar e testar" para chegar em soluções ótimas ou quase ótimas, sem grandes limitações. (CARVALHO, 2021).

Outra técnica computacional para otimizações matemáticas que se destaca por sua simplicidade de implementação é o Scipy.optimize.minimize o qual permite encontrar os valores de mínimos, máximos ou zeros de uma função (SCIPY-LECTURES, 2020).

Para a aplicação dos métodos citados na otimização de uma fábrica de bebidas fazse necessário inicialmente ter o conhecimento dos processos envolvidos e da gama de produtos fabricados. Em seguida propõem-se então soluções que satisfazem de forma eficiente as demandas da empresa. (PESSANHA; ALVARENGA; ARICA, 2015).

O objetivo deste trabalho foi otimizar o estoque de uma empresa de bebidas não alcoólicas, utilizando de dados fornecidos pela empresa, como seu histórico de vendas e controles de estoque. Com a aplicação dos métodos de otimização e baseado nos dados fornecidos, foi possível implementar um algoritmo que analisa a previsão de vendas e gerencia o estoque, sem que a metodologia seja invasiva ao funcionamento das máquinas da fábrica.

Levando em conta que a variação de vendas dos produtos funciona de forma sazonal, pode-se estimar uma produção necessária baseada nas vendas de meses anteriores e assim evitar que ocorram falta de produtos nos estoques ou também um excesso de produção, ambos prejudiciais ao orçamento da empresa.

As vantagens da aplicação do método utilizado neste trabalho são o baixo custo da implementação proposta, baseada em linguagem Python, um software livre. Além disso não houve necessidade de visitas presenciais à empresa, por utilizar apenas os bancos de dados históricos da empresa.

Como resultados deste trabalho têm-se a diminuição de custo gerada indiretamente por um melhor controle de estoques, melhorando sua organização de produção, diminuindo horas de trabalho e de setup de maquinário. 


\section{PROCEDIMENTOS METODOLÓGICOS}

Em busca de uma solução mais próxima de um ótimo nominal para o problema proposto neste trabalho, todo o funcionamento da linha de produção de uma indústria de bebidas não alcoólicas reais, localizada no estado de São Paulo, foi analisado. Foi feito a modelagem matemática do controle de estoque da empresa baseado em históricos de vendas. Outros parâmetros foram também mapeados como a capacidade máxima de produção, a qual está ligada à quantidade de garrafas que a máquina responsável por moldar as garrafas pets (sopradora) conseguem produzir. Foi verificado este processo como sendo o mais crítico da fábrica, sendo então utilizado para encontrar a função objetivo e suas restrições, descritas a seguir, as quais são utilizadas pelos métodos de otimização. Buscando o mínimo global de uma função objetivo limitado por restrições é possível encontrar a melhor solução possível e real para o problema.

\section{Restrições}

Com os dados de limite da produção, estoques limiares e vendas previstas foi possível encontrar equações matemáticas que representam as restrições reais da fábrica. No equacionamento das restrições foram criadas duas variáveis dependentes, denominadas rest 1 e rest 2 , definidas de maneira a ser implementadas por cada um dos dois métodos estudados.

Para cada rotina de código, foram criadas listas de quantidades de produto. Essas listas possuem os valores previstos de venda $(V p)$, que são baseados nas vendas do ano anterior. Em seguida são calculados os demais parâmetros de entrada dos algoritmos dados pelo estoque inicial ( $E i$ - equação 1), estoque desejado ( $E d$ - equação 2) e limiar de estoque ( $L$ - equação 3 ), respectivamente definidos proporcionalmente à $V p$ e conforme esperado pela equipe gestora da empresa.

$$
\begin{aligned}
& E i=V p \times 0.6 \\
& E d=V p \times 0.7 \\
& L=V p \times 0.1
\end{aligned}
$$

Nas restrições do algoritmo de minimização Scipy.optimize.minimize foram utilizadas funções de desigualdade, as quais devem ser não negativas, ou seja, conforme definido pela biblioteca do método, os valores das variáveis de restrição devem ser maiores ou iguais a zero. A partir dos dados de previsão de venda para cada produto, contidos no vetor $V p$, é possível calcular a quantidade " $\mathrm{x"} \mathrm{de} \mathrm{cada} \mathrm{produto} \mathrm{"j"} \mathrm{que} \mathrm{deverá} \mathrm{ser} \mathrm{produzida} \mathrm{para}$ suprir a demanda.

$$
\begin{aligned}
& E f[j]=E i[j]-V p[j]+x[j] \\
& \operatorname{rest} 1[j]=E f[j]-L[j] \\
& \text { rest2 }=P_{-} \max -n p . \operatorname{sum}(x)
\end{aligned}
$$

No qual "Ef", equação 4, é o estoque final do ciclo de produção, dado pela diferença do estoque inicial mais a produção menos a previsão de venda; $P_{-}$max é a produção máxima real da fábrica; e np. $\operatorname{sum}(x)$ (comando da biblioteca Numpy) é a soma da produção de todos os produtos. Na primeira restrição, equação 5 , a diferença do estoque final e o 
limiar do estoque deve ser sempre maior ou igual a zero. Na segunda restrição, equação 6, a soma de produção de todos os produtos deve ser menor ou igual ao limite de produção da fábrica.

Para o método de Genetic Algorithm, as restrições se baseiam nas mesmas informações, porém enquanto a primeira restrição é ainda obtida pela equação 5 , a segunda restrição é obtida pela equação 7 , de maneira a cumprir com as exigências de funcionamento do algoritmo, neste caso:

$$
\text { rest2 }=\operatorname{np} \cdot \operatorname{sum}(x)-P_{-} \max
$$

Os algoritmos buscam otimizar a diferença entre o estoque final e o desejado variando a quantidade de produtos produzidos, sendo que a resposta deve ser buscada dentro de um intervalo delimitado pelas restrições de desigualdade, até convergir para o melhor resultado possível. Para que as restrições criadas façam com que o sistema convirja para um resultado plausível com o real, foi criado um sistema de penalidades, no qual as respostas encontradas que estejam fora dos limites das restrições são igualadas a um valor elevado. Neste caso, $10^{10}$ foi a penalidade escolhida para que o usuário saiba que a resposta recebida foi penalizada, ou seja, o código descarta a solução e gera uma nova interação para buscar uma solução otimizada.

Tendo definido o sistema de equações de restrições para os dois algoritmos, pode-se partir para a modelagem da função objetivo.

\section{Função objetivo}

A função objetivo é calculada a partir da mesma base de dados utilizada nas restrições, ou seja, utiliza da formulação de Estoque final (equação 4) e Estoque desejável (equação 2) para otimizar a diferença entre os dois parâmetros.

$$
\text { Var }=n p \cdot \operatorname{sum}(n p \cdot a b s(E d[j]-E f[j]))
$$

O problema se resume a uma única função objetivo denominada $V a r$, conforme equação 8, a qual é obtida pela soma, para todos os produtos fabricados, dos módulos das diferenças entre o estoque desejado e o estoque final. A mesma formulação é utilizada para os dois algoritmos. Portanto o algoritmo irá buscar a produção $x$ [j] que minimize o valor de Var, respeitando as limitações implementadas nas restrições.

Caso ocorra de o sistema não convergir, é possível alterar os inputs de número de iterações e tamanho da população para que haja mais possibilidades de o algoritmo encontrar uma resposta que convirja. Como, por exemplo, quando o número de iterações é muito baixo, não há a possibilidade de o código convergir para um resultado otimizado.

\section{RESULTADOS E DISCUSSÃO}

\section{Resultados Computacionais}

Nesta seção do trabalho serão apresentados os resultados obtidos na implementação dos dois métodos de otimização (Genetic algorithm e Scipy.optimize.minimize).

Foram utilizados respectivamente as seguintes bibliotecas do Python: 
1- geneticalgorithm ${ }^{1}$;

2- scipy.optimize com o método SLSQP 2.

Os valores dos parâmetros de setup foram escolhidos a partir de uma análise de sensibilidade para cada um dos algoritmos. Visto que o objetivo deste trabalho não é discutir a influência dos parâmetros, e sim os resultados do estudo de caso abordado, são apresentados os parâmetros e resultados do caso, dentre os que foram simulados, que se mostrou o mais otimizado. Mais otimizado é considerado o caso que possui menor valor da função objetivo (equação 8). Isso quer dizer que a escolha dos parâmetros foi independente do tempo de processamento. Os resultados do tempo de processamento são apresentados apenas com o objetivo de comparar o desempenho computacional dos métodos, tendo como referência as simulações em um computador Intel Core i7 com 8 Gb de memória RAM.

Para o Algoritmo Genético (Genetic Algorithm), os parâmetros de inicialização do código foram determinados como demonstram as equações de 9 a 13.

$$
\begin{aligned}
& n=22 \\
& E a=n p \cdot \operatorname{zeros}(n) \\
& \operatorname{Var}=n p \cdot \operatorname{zeros}(n) \\
& r e s t 1=n p \cdot \operatorname{zeros}(n) \\
& r e s t 2=n p \cdot \operatorname{zeros}(n)
\end{aligned}
$$

Os parâmetros internos escolhidos no Algoritmo genético para entregar a resposta mais otimizada dentro da capacidade de processamento do computador utilizado foram escolhidos da forma como está apresentado nas equações de 14 a 21.

'max_num_iteration': 800

'population_size': 800

'mutation_probability': 0.2

'elit_ratio': 0.01

'crossover_probability': 0.3

'parents_portion': 0.3

crossover_type': 'uniform'

'max_iteration_without_improv': None

Para o algoritmo Scipy.optimize.minimize, foram utilizados os mesmos parâmetros iniciais determinados para o Algoritmo Genético e os parâmetros internos foram definidos dentro das opções dadas pelo método "SLSQP" como é apresentado nas equações 22 e 23.

'type': 'ineq', 'fun': restricao1

'type': 'ineq',' fun': restricao 2

\footnotetext{
${ }^{1}$ Disponível em: https://pypi.org/project/geneticalgorithm/

${ }^{2}$ Disponível em: https://docs.scipy.org/doc/scipy/reference/optimize.minimize-slsqp.html
} 
Revista Brasileira de Ciência, Tecnologia e Inovação

As informações utilizadas foram coletadas no banco de dados da empresa e informações técnicas dos responsáveis da produção. Este estudo leva em conta todas as limitações reais da fábrica analisada, para que não haja divergências no resultado obtido neste trabalho e as possibilidades de implementação na fábrica. Para o desenvolvimento deste trabalho considerou-se as vendas do mês de outubro de 2020 como base de dados inicial e a produção total de 430.000 garrafas produzidas em funcionamento normal das máquinas.

A Tabela 1 fornece a quantidade de garrafas vendidas de cada produto produzido na empresa no período do mês de outubro. Com estes dados é possível obter os valores de estoque inicial, estoque desejado e limiar de estoque para aplicar nas formulações do código.

Tabela 1. Quantidade de garrafas vendidas de cada produto no mês de outubro.

\begin{tabular}{clc}
\hline & PRODUTO & QUANTIDADE DE GARRAFAS \\
\hline 1 & Guaraná 2L & 37674 \\
2 & Gipps guaraná 2L & 16872 \\
3 & Laranja 2L & 18702 \\
4 & Limão 2L & 9186 \\
5 & Citrus 2L & 12474 \\
6 & Cola 2L & 37980 \\
7 & Água c/ gás 2L & 9180 \\
8 & Água c/ gás limão 1L & 4716 \\
9 & Água c/ gás 510ml & 42120 \\
10 & Água c/ gás limão 510ml & 97380 \\
11 & Água c/ gás maçã 510ml & 30876 \\
12 & Guaraná 250ml & 21732 \\
13 & Laranja 250ml & 4620 \\
14 & Maçã 2L & 38586 \\
15 & Uva 2L & 11406 \\
16 & Uva 250ml & 4884 \\
17 & Cola 250ml & 6756 \\
18 & Cola 1L & 2592 \\
19 & Guaraná 1L & 6660 \\
20 & Maçã 1L & 11784 \\
21 & Maça 250ml & 17652 \\
22 & Energético k10 & 1800 \\
\hline
\end{tabular}

A partir dos dados da Tabela 1 é possível encontrar também a produção ideal, baseada no estoque inicial e a previsão de venda, que seriam de 490.195 garrafas. Esta produção ideal indica qual deveria ser a produção de cada item de maneira a resultar em uma quantidade em estoque igual à desejada. No entanto, com a produção máxima possível pela fábrica de 430.000 garrafas/mês, é necessário que haja uma otimização da produção para encaixar a melhor quantidade de produção de cada produto e assim minimizar a diferença entre estoque desejado e estoque final. 
Para o algoritmo Scipy.optimize.minimize e para o Genetic Algorithm, foram encontrados os resultados de produção necessária para otimizar o estoque apresentados na Tabela 2.

Pode-se observar na Tabela 2 que as produções necessárias encontradas pelos dois algoritmos buscam, de maneiras diferentes, encontrar o estoque final mais próximo do estoque desejado. Apesar do algoritmo Scipy.optimize.minimize ter um tempo de processamento de cerca de 1 segundo, observa-se que o Genetic Algorithm, no qual o tempo de processamento é de cerca de 1 minuto e meio, tem maior eficácia, pois encontra não apenas um resultado mais otimizado, e sim um resultado com melhor aplicabilidade real, se adaptando à produção necessária de cada produto.

Na Tabela 3 é apresentado um comparativo dos valores de estoque desejado com os valores de estoques finais resultantes da aplicação de ambos os métodos.

Tabela 2. Produção otimizada de cada produto.

\begin{tabular}{clcc}
\hline & PRODUTO & SCIPY MINIMIZE & GA \\
\hline 1 & Guaraná 2L & 41441 & 29334 \\
2 & Gipps guaraná 2L & 18559 & 15725 \\
3 & Laranja 2L & 20572 & 17562 \\
4 & Limão 2L & 10104 & 7238 \\
5 & Citrus 2L & 13721 & 12816 \\
6 & Cola 2L & 41777 & 35068 \\
7 & Água c/ gás 2L & 10097 & 8121 \\
8 & Água c/ gás limão 1L & 5187 & 3857 \\
9 & Água c/ gás 510ml & 46331 & 36967 \\
10 & Água c/ gás limão 510ml & 46922 & 106227 \\
11 & Água c/ gás maçã 510ml & 33963 & 31629 \\
12 & Guaraná 250ml & 23905 & 21524 \\
13 & Laranja 250ml & 5081 & 4302 \\
14 & Maçã 2L & 42444 & 39519 \\
15 & Uva 2L & 12546 & 12444 \\
16 & Uva 250ml & 5372 & 3176 \\
17 & Cola 250ml & 7431 & 5743 \\
18 & Cola 1L & 2851 & 1802 \\
19 & Guaraná 1L & 7325 & 4856 \\
20 & Maçã 1L & 12962 & 11401 \\
21 & Maça 250ml & 19417 & 19403 \\
22 & Energético k10 & 1979 & 1264 \\
\hline & Total & 429987 & 429978 \\
\hline
\end{tabular}


Revista Brasileira de Ciência, Tecnologia e Inovação

Tabela 3. Comparação dos Estoques finais otimizados com o Estoque desejado.

\begin{tabular}{clccc}
\hline PRODUTO OUTUBRO & $\begin{array}{c}\text { ESTOQUE } \\
\text { DESEJADO }\end{array}$ & $\begin{array}{c}\text { ESTOQUE FINAL } \\
\text { SCIPY.MINIMIZE }\end{array}$ & $\begin{array}{c}\text { ESTOQUE FINAL } \\
\text { GENETIC ALGORITHM }\end{array}$ \\
\hline 1 & Guaraná 2L & 26371 & 26371 & 14265 \\
2 & Gipps guaraná 2L & 11810 & 11810 & 8977 \\
3 & Laranja 2L & 13091 & 13091 & 10081 \\
4 & Limão 2L & 6430 & 6430 & 3564 \\
5 & Citrus 2L & 8731 & 8731 & 7828 \\
6 & Cola 2L & 26586 & 26585 & 19876 \\
7 & Água c/ gás 2L & 6426 & 6425 & 4449 \\
8 & Água c/ gás limão 1L & 3301 & 3301 & 1970 \\
9 & Água c/ gás 510ml & 29484 & 29483 & 20119 \\
\hline 10 & Água c/ gás limão 510ml & 68166 & 7970 & 67275 \\
\hline 11 & Água c/ gás maçã 510ml & 21613 & 21613 & 19279 \\
\hline 12 & Guaraná 250ml & 15212 & 15212 & 12831 \\
13 & Laranja 250ml & 3234 & 3233 & 2454 \\
\hline 14 & Maçã 2L & 27010 & 27010 & 24085 \\
15 & Uva 2L & 7984 & 7984 & 7882 \\
\hline 16 & Uva 250ml & 3418 & 3418 & 1222 \\
\hline 17 & Cola 250ml & 4729 & 4729 & 3040 \\
\hline 18 & Cola 1L & 1814 & 1814 & 765 \\
\hline 19 & Guaraná 1L & 4662 & 4661 & 2192 \\
\hline 20 & Maçã 1L & 8248 & 8248 & 6687 \\
21 & Maça 250ml & 12356 & 12356 & 12342 \\
\hline 22 & Energético k10 & 1260 & 1259 & 544 \\
\hline & Total & 311936 & 251734 & 215727 \\
\hline & & & & \\
\hline
\end{tabular}

Na Tabela 3 é verificado que o algoritmo Scipy.optimize.minimize busca a melhor otimização de produção necessária para cada produto, mesmo que acabe faltando espaço de produção para um dos produtos. Já para o Genetic Algorithm, pode-se observar uma melhor interpretação da necessidade da fábrica, fazendo com que a produção possa ser menor em cada produto, mas sempre suprindo uma prioridade de produção, ou seja, buscando um resultado mais plausível com a realidade.

Verifica-se na Tabela 2 que a produção total de acordo com cada algoritmo está próxima ao limite da fábrica de 430 mil garrafas, portanto não seria possível aumentar a produção. Porém o objetivo é minimizar a diferença entre o estoque desejado e o estoque final, conforme Tabela 3. Ao analisar a Tabela 3 observa-se que o valor total de acordo com cada algoritmo está abaixo do desejado, porém não é possível aumentar a produção conforme visto na Tabela 2. Caso a produtividade máxima da fábrica fosse maior essa diferença do total dos estoques finais para o desejado seria menor, sendo que o algoritmo busca otimizar exatamente para que a diferença seja a mínima possível, levando em conta as restrições. A quantidade de produtos fabricados somado ao estoque inicial atende ainda a previsão de venda da Tabela 1, caso contrário o estoque final seria negativo, o que não foi observado. A limitação de produção máxima afeta apenas o estoque final, não se tratando, portanto, de uma perda de vendas, e sim um controle de estoque levando em conta não apenas a quantidade de garrafas produzidas, mas também o estoque inicial e a 
Revista Brasileira de Ciência, Tecnologia e Inovação

previsão de vendas, obtidos pelo histórico de vendas de outubro apresentado na Tabela 1. Por fim a restrição de estoque final acima do limiar também foi atendida por ambos os algoritmos.

\section{Comparativo entre resultados otimizados de estoque final em outubro e vendas reais de novembro}

Após a obtenção dos resultados otimizados pode-se comparar os dados de estoque final do mês de outubro previsto por ambos os métodos, ou seja, o estoque inicial do mês de novembro, com os valores reais de vendas realizadas pela fábrica no mês de novembro de 2020, conforme Figura 1. Desta forma é possível compreender de maneira ainda mais aprofundada o comportamento de cada algoritmo e assim definir se os algoritmos seriam confiáveis em gerir a produção de maneira compatível com as vendas do mês seguinte. Analisando a Figura 1 pode-se verificar que o Genetic Algorithm resulta em um estoque mais seguro, isto é, garantindo a demanda de novembro mesmo em situações onde as vendas de um certo produto saiam da média. O mesmo não é observado na curva do Scipy.optimize.minimize, onde o gráfico é extremamente fiel às vendas de Novembro em quase todos os produtos, porém não consegue se adaptar e atender as situações onde as vendas têm um comportamento diferente do esperado, como o produto 10.

Figura 1. Gráfico comparativo entre estoque final otimizado de outubro e vendas de novembro.

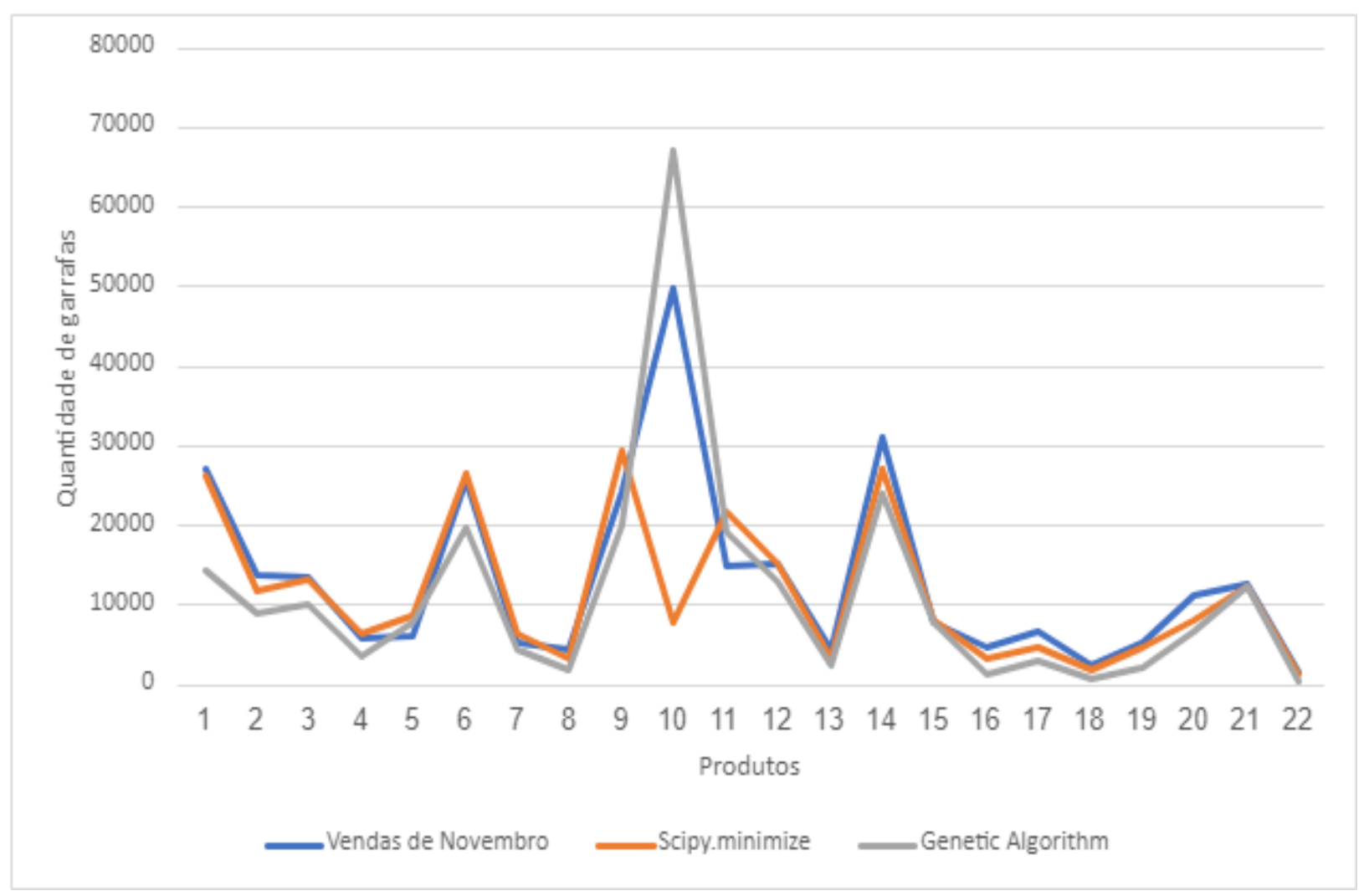

Conclui-se que o Genetic Algorithm apresenta um melhor resultado pois atende melhor em casos que não se tem uma uniformidade nas vendas entre os produtos. 
Revista Brasileira de Ciência, Tecnologia e Inovação

Novamente destaca-se que a Figura 1 se trata de uma análise de estoque inicial com vendas previstas, ou seja, não se trata de dizer que o estoque não atenderá as vendas de novembro. O que é observado na Figura 1 é que o estoque final de outubro otimizado pelo Genetic Algorithm por si só quase supriria as vendas de novembro, sendo considerado um estoque seguro. $O$ algoritmo pode então ser novamente empregado para prever a produção do mês de novembro que suprirá a diferença do estoque inicial, as vendas previstas e ainda minimizar a diferença para que no final de novembro o estoque final seja o mais próximo possível ao desejado, dentro das restrições. O processo se repete para os meses consecutivos.

Destaca-se que a diferença no comportamento do produto 10 surge ao comparar o resultado do Scipy.optimize.minimize com as vendas futuras, ou seja, no momento da otimização os dados das vendas de novembro não são conhecidos. Portanto, não se trata de uma divergência do método e sim de duas interpretações diferentes de possíveis distribuições da produção. Os resultados do Genetic Algorithm resultam em um comportamento com menor diferença para o produto 10 pois no mês de Outubro a produção otimizada foi maior que a do Scipy.optimize.minimize, sendo, portanto, ambas soluções válidas e ambas atendem as restrições impostas.

\section{CONCLUSÕES}

Neste trabalho foram utilizados dois métodos de otimização computacionais para a resolução de um problema de controle de estoque de uma empresa de bebidas. Ambos os algoritmos implementados obtiveram resultados otimizados, porém com aplicabilidades diferentes.

Visto que não é de conhecimento dos autores nenhum estudo de caso semelhante apresentado em trabalhos científicos presentes na literatura, foram utilizados neste trabalho os dados reais de uma fábrica e assim analisados os resultados obtidos pelos modelos de otimização propostos.

Comparativamente, o algoritmo Scipy.optimize.minimize tem seu tempo de processamento dezenas de vezes mais rápido. Porém sua desvantagem está no fato de não compreender a distribuição de cada produto na produção geral, podendo fazer com que um produto de alta demanda tenha sua produção debilitada.

O Genetic Algorithm por sua vez, consegue encontrar uma produção suficientemente otimizada e dentro dos parâmetros de demanda de produtos, pois analisa diversas possíveis respostas e busca a que se encaixa melhor com a distribuição de cada produto nas vendas gerais. Porém, para que haja eficiência no código, é necessário que o número de iterações e o tamanho da população do código sejam grandes, ocasionando em um maior tempo de processamento.

Conclui-se que o Genetic Algorithm apresenta melhores resultados, pois atende melhor em casos que não se tem uma uniformidade nas vendas entre os produtos, garantindo um estoque final mais seguro para atender o mês seguinte.

Com as soluções obtidas é possível otimizar diferentes necessidades do mercado, aumentando a rentabilidade do negócio e sem que haja grandes custos operacionais.

A sugestão dos autores para trabalhos futuros é a aplicação da metodologia proposta ao longo de um ano de produção da fábrica de maneira a identificar possíveis influências de algum parâmetro não mapeado e assim poder aprimorar o modelo utilizado. 
RBCII

\section{REFERÊNCIAS}

CARVALHO, A. P. L. F. Algoritmos genéticos. Disponível em:

https://sites.icmc.usp.br/andre/research/genetic/. Acesso em: 10 jan. 2021.

MALAQUIAS, N G. L. Uso dos algoritmos genéticos para a otimização de rotas de distribuição. 2006. 113p. Tese (Mestrado em Engenharia Elétrica) - Universidade Federal de Uberlândia, Faculdade de Engenharia Elétrica, Uberlândia, MG, 2006.

PAGLIARUSSI, M. S.; MORABITO, R; SANTOS, M. O. Otimização da programação da produção de bebidas à base de frutas por meio de modelos de programação inteira mista. Scientific Electronic Library Online - Scielo, p. 1, dezembro 2016. DOI: https://doi.org/10.1590/0104-530X2288-15. Disponível em:

https://www.scielo.br/j/gp/a/XM8DcbSWFGDHCTPfHWJWrCB/?lang=pt. Acesso em: 09 jan. 2021.

PESSANHA, L. P. M.; ALVARENGA, R. L.; ARICA, G. G. M. Modelagem e resolução do problema integrado de dimensionamento e sequenciamento da produção: Caso de uma pequena empresa de produtos de limpeza. In: XXXV Encontro nacional de engenharia de produção. Fortaleza, CE, 2015.

SCIPY-LECTURES. Mathematical optimization: finding minima of functions.

Disponível em: https://scipy-lectures.org/advanced/mathematical_optimization/. Acesso em: 23 dez. 2020.

SEBRAE. Micro e pequenas empresas geram 27\% do PIB do Brasil. Disponível em: https://www.sebrae.com.br/sites/PortalSebrae/ufs/mt/noticias/micro-e-pequenasempresas-geram-27-do-pib-do-brasil,ad0fc70646467410VgnVCM2000003c74010aRCRD. Acesso em: 09 jan. 2021.

Recebido em: 12/04/2021

Aprovado em: 04/07/2021 